\title{
Associação entre distúrbios reprodutivos e anticorpos anti-Brucella sp em cães atendidos em clínicas particulares da cidade de Maceió-AL
}

\section{Agglutinins against Brucella canis and Brucella abortus and risk factors for Brucella canis infected dogs examined in private veterinary clinics in the city of Maceió-Alagoas}

\author{
Wagnner José Nascimento Porto, ${ }^{\star}$ José Wilton Pinheiro Junior, ${ }^{\star *}$ Rinaldo Aparecido Mota***
}

\begin{abstract}
Resumo
Objetivou-se com este estudo determinar a freqüência de aglutininas anti-B. canis e Brucella abortus, além de identificar possíveis fatores de riscos associados à infecção em cães atendidos nas clínicas veterinárias da Cidade de Maceió, Alagoas. Foram utilizados 90 animais, sendo 28 machos e 62 fêmeas de diferentes raças e faixa etária variável. Para pesquisa de aglutininas anti-Brucella canis utilizaram-se as provas de imunodifusão em gel de agarose (IDGA) e imunodifusão com 2mercaptoetanol (IDGA/2-ME) e para pesquisa de aglutininas anti-Brucella abortus utilizou-se o teste do Antígeno Acidificado (AAT). Dos 90 animais analisados quatro $(4,4 \%)$ foram positivos no IDGA, dos quais três $(75,0 \%)$ foram positivos ao IDGA/2$\mathrm{ME}$, enquanto um $(25,0 \%)$ foi negativo. Ao teste do AAT nenhum animal foi soro-reagente. A análise de concordância entre os testes utilizados foi $K=0,851$. Não foi observada associação significativa para as variáveis faixa etária $(p=0,426)$ e sexo $(p=0,678)$ e apenas a variável raça apresentou associação significativa para infecção por Brucella canis com resultado do odds ratio 0,04 $(p=0,025 ;$ IC $95 \% 0,00 ; 1,01)$. Os resultados obtidos neste estudo demonstram que a infecção por Brucella canis ocorre na Cidade de Maceió-AL e que medidas de controle e profilaxia devem ser empregadas para evitar a disseminação do agente para criatórios livres da doença.
\end{abstract}

Palavras-chave: brucelose, epidemiologia, animais de companhia.

\begin{abstract}
This study aimed to determine the frequency of agglutinins against Brucella canis and Brucella abortus, and to identify possible risk factors associated with infection in dogs examined in private veterinary clinics in the city of Maceió-Alagoas. For the purpose of this study we used 90 animals: 28 males and 62 females of various breeds and ages. Immune Diffusion in Gel Agar (IDGA) and immune diffusion with 2-Mercaptoethanol (IDGA/2-ME) were used to investigate the presence of agglutinins against $B$. canis, and the Acidified Antigen Test (AAT) was used to investigate agglutinins against $B$. abortus (AAT). Out of 90 animals four (4.4\%) had positive IDGA; with three (75.0\%) testing positive on the IDGA/2-ME and one was negative on the IDGA/2-ME. No animal tested positive on the AAT. The concordance analysis between the tests was $K=0.851$. No significant association was noticed for age group $(p=0,426)$ and gender $(p=0,678)$. The only variable with significant association for $B$. canis infection was breed, with 0.04 odds ratio $(p=0.025$; IC $95 \% 0.00 ; 1.01)$. The results of this study show that $B$. canis infection is present in the city of Maceió-AL, and that control and preventive measures must be enforced to prevent dissemination into disease-free populations.
\end{abstract}

Keywords: brucellosis, epidemiology, companion animals.

\section{Introdução}

A urbanização e mudanças sociais da população humana nas últimas décadas favoreceram o aumento da população canina nos países em desenvolvimento. Esse aumento, associado às relações sentimentais/emocionais do homem com esta espécie tem implicações nos problemas de saúde pública, pois o animal pode ser responsável pela transmissão de várias zoonoses, entre elas, a brucelose (Souza et al., 2002).
A brucelose animal assume importância significativa entre as doenças ou infecções naturalmente transmissíveis dos animais ao homem, e vice-versa, na medida em que afeta a saúde animal, a economia da produção pecuária, a saúde pública e a disponibilidade de proteína animal para as necessidades da população (Acha e Syfres, 1989).

A infecção de cães por Brucella canis é relatada em praticamente todos os países, com prevalência variável segundo a

\footnotetext{
* Professor de Parasitologia, Universidade Estadual de Alagoas, Av. Governador Luiz Cavalcante, s/n, Arapiraca, AL, CEP 57312-270

** Professor Adjunto da Unidade Acadêmica de Garanhuns da Universidade Federal Rural de Pernambuco

*** Professor Associado do Departamento de Medicina Veterinária da Universidade Federal Rural de Pernambuco, R. Dom Manoel de Medeiros, $\mathrm{s} / \mathrm{n}$ - Dois Irmãos, Recife, PE. CEP 52171-900 rinaldo.mota@hotmail.com
} 
região e o método de diagnóstico empregado, podendo-se afirmar que apresenta distribuição mundial (Carmichael e Greene, 1993). Quanto às alterações clínicas compatíveis com a brucelose canina, os sintomas e lesões estão relacionados com o aparelho reprodutor de machos e fêmeas (Gomes et al., 1999) que tenham atingido a maturidade sexual (Carmichael e Greene, 1993).

O diagnóstico clínico da infecção é bastante difícil devido à ausência de sinais específicos e a maioria dos cães serem assintomáticos (Cavalcanti et al., 2006). Em função da dificuldade e da baixa especificidade do diagnóstico clínico, a confirmação da brucelose canina deve ser realizada por métodos laboratoriais (Minharro et al., 2005). Ainda segundo esses autores, o isolamento e a identificação da $B$. canis é um método de alta especificidade diagnóstica, pois demonstra o agente etiológico da doença, mas sua sensibilidade pode ser baixa em detrimento de vários fatores como eliminação intermitente da bactéria, material mal coletado e mal conservado, além da utilização de antibióticos.

Dessa forma, os testes sorológicos representados pelas provas de soroaglutinação lenta, rápida e a imunodifusão em gel de agarose (IDGA) são os métodos freqüentemente utilizados no diagnóstico da doença.

Considerando a importância dessa enfermidade, além da escassez de dados na cidade de Maceió, Alagoas, objetivouse com esse estudo determinar a freqüência de aglutininas anti- $B$. canis e $B$. abortus em cães, além de identificar possíveis fatores de riscos associados à infecção.

\section{Material e métodos}

Foram utilizados 90 animais, sendo 28 machos e 62 fêmeas de diferentes raças e faixa etária variável, atendidos em clínicas veterinárias da cidade de Maceió, Alagoas. Realizou-se anamnese para verificar a presença ou ausência de sinais de distúrbios reprodutivos. Destes animais, 31 apresentavam sinais clínicos sugestivos de brucelose e 59 encontravam-se clinicamente sadios.

As amostras foram obtidas por meio da venopunção da cefálica e foram armazenadas em tubos de ensaio, identificados e encaminhados ao Laboratório de Doenças Infecto-contagiosas da Universidade Federal Rural de Pernambuco; para obtenção do soro, centrifugou-se por dez minutos a 900 giros e estes foram armazenados em microtubos e submetidos ao congelamento até o momento das análises.

Para realização das provas de IDGA e IDGA/2-ME utilizou-se antígeno de lipopolissacarídeos e proteínas de Brucella ovis (amostra Reo 198) fornecido pelo Instituto de Tecnologia do Paraná - TECPAR. Na preparação do gel utilizou-se agarose (DIFCO) a $0,5 \%$, dissolvido em solução salina a $10 \% \mathrm{com}$ tampão de Sorensen, ajustando-se o pH final da solução para 8,3. A distribuição do gel, perfuração dos poços, leitura em placas de Petri descartáveis e acondicionamento em câmara úmida, obedeceram às especificações do fabricante do antígeno.

Para confirmação da infecção por $B$. canis os soros foram submetidos à prova IDGA/2-ME, realizando-se tratamento com $0,2 \mathrm{M}$ de 2 -Mercaptoetanol. Dessa forma, ao volume de $100 \mu \mathrm{L}$ de soro foram adicionadas $100 \mu \mathrm{L}$ da solução de 2-ME a 0,2M, permanecendo em reação durante 30 minutos (KEID, 2001). Após esse período, $25 \mu \mathrm{L}$ dos soros tratados foram submetidos à IDGA seguindo o protocolo preconizado pelo TECPAR.

Para pesquisa de aglutininas anti-Brucella abortus os soros foram submetidos ao teste do AAT de acordo com Alton et al. (1988).

A análise para comparação dos testes sorológicos IDGA e IDGA/2-ME foi baseada no estudo de concordância entre os métodos por meio do coeficiente Kappa de acordo com Truesfield (1986).

Para o cálculo da freqüência dividiu-se o número de animais sorologicamente positivos ao teste IDGA/2-ME pelo número de animais amostrados, utilizando-se análise estatística descritiva por meio de distribuições absoluta e relativa. Para o estudo da associação entre a soropositividade e as variáveis analisadas (faixa etária, raça, sexo), utilizou-se estatística inferencial por meio do teste Exato de Fisher e os intervalos foram obtidos com confiabilidade de $95 \%$. O nível de significância utilizado na decisão dos testes estatísticos foi de $5 \%$. O programa utilizado para a obtenção da análise estatística foi o Epilnfo versão 6.02 (Dean et al., 1994)

\section{Resultados e discussão}

Dos 90 animais analisados, quatro $(4,4 \%)$ foram positivos ao teste do IDGA, e destes, três $(75,0 \%)$ foram positivos ao IDGA/ 2-ME, enquanto que uma $(25,0 \%)$ amostra foi negativa. A análise de concordância entre os testes utilizados nesse estudo encontra-se na Tabela 1. Nenhum animal foi reagente ao teste do AAT para a pesquisa de anticorpos anti-Brucella abortus.

Tabela 1: Análise de concordância entre as provas Imunidifusão em Gel de Agarose (IDGA) e Imunidifusão em Gel de Agarose tratadas com 2-Mercaptoetanol (IDGA/2ME) em cães atendidos em clínicas particulares na cidade de Maceió, Alagoas

\begin{tabular}{lccccc}
\hline \multirow{2}{*}{ IDGA } & \multicolumn{2}{c}{ IDGA/2-ME } & Total & Valor Kappa \\
\cline { 2 - 3 } & & Positivo & Negativo & & \\
\hline Positivo & 03 & 01 & 04 & 0,851 \\
Negativo & - & 86 & 86 & \\
\hline Total & 03 & 87 & 90 & \\
\hline
\end{tabular}

De acordo com revisão realizada por Almeida et al. (2004) os índices de prevalência de animais sororreagentes para Brucella abortus são baixos. A não-presença de infecção pela $B$. abortus nesse estudo pode ser justificada uma vez que não possuíam contato com animais de produção.

Os resultados da freqüência de animais sororreagentes observados neste estudo para aglutininas anti-Brucella canis foram superiores aos descritos em Belo Horizonte, 1,3\% (Godoy et al., 1977) e na Microrregião da Serra de Botucatu, $1,77 \%$ (Moraes et al., 2002). Porém, foram inferiores aos relatados em São Paulo 9,1\% em cães de canil e 7,5\% em cães errantes (Larson et al., 1981), 7,5\% (Cortes et al., 1988); 9,51\% em Santana de Parnaíba (Azevedo, 2002); em Porto 
Alegre, 11,97\% (Wald e Fernandes, 1976/1977); no Rio de Janeiro e em Niterói, 29,4\% e 19,2\%, respectivamente (Maia et al., 1999); em Uruguaiana, 72,7\% (Vargas et al., 1996) e Recife, 16,6\% (Numeriano et al., 1999).

As variações encontradas podem ser explicadas provavelmente devido à utilização de métodos sorológicos diferentes que apresentam sensibilidade e especificidade diferentes (Nicoletti e Chase, 1988) e ao tipo de amostra estudada, bem como pela presença de animais no início de infecção, onde os anticorpos podem ainda não ser detectados no IDGA (Carmichael e Greene, 1993).

Comparando-se os resultados nas provas utilizadas, observou-se uma diferença na freqüência de animais sororreagentes. Resultados inferiores foram relatados por Keid (2001), $44,8 \%$ (IDGA) e 6,25\% (IDGA/2-ME) e Azevedo (2002) que encontrou 48,75\% (IDGA) e 18,75\% (IDGA/2-ME). Essas diferenças observadas podem ser justificadas pela ocorrência de reações inespecíficas quando da utilização da IDGA, pois os antígenos da parede celular (LPS) de algumas bactérias Gram-negativas apresentam reação cruzada com a Brucella canis (Johnson e Walker, 1992; Carmichael e Greene, 1993). Podem ocorrer também porque alguns animais sororreagentes na IDGA poderiam estar na fase aguda da doença onde são produzidos anticorpos da classe lgM (Carmichael e Kenney, 1970) que são desnaturadas pelo 2-ME, portanto, o teste IDGA + 2ME detecta apenas animais na fase crônica da doença. Outra possibilidade seria a desnaturação das IgM procedentes de reações inespecíficas (Greene e George, 1984; Johnson e Walker, 1992; Carmichael e Shin, 1996), reduzindo também o número de animais reagentes quando se utiliza o 2-ME.

Os principais distúrbios reprodutivos encontrados este estudo foram: infertilidade $(60,0 \%)$ e perda da libido $(40,0 \%)$ nos machos; aborto $(33,3 \%)$, número reduzido de filhotes $(23,8 \%)$, infertilidade $(19,1 \%)$, nascimento de filhotes debilitados que vieram a óbito na primeira semana de vida $(14,3 \%)$, natimortos e corrimento vaginal $(9,5 \%)$, má-formação fetal, pseudociese e piometra $(4,8 \%)$, nas fêmeas. Quando se analisaram os animais que apresentavam ou não sinais clínicos sugestivos da brucelose canina, observou-se que 6,5\% destes mostraram-se positivos à prova de IDGA, enquanto nos animais que não apresentavam sintomas, apenas 3,4\% foram considerados positivos.

Quando se aplicou o teste estatístico, a associação entre distúrbios reprodutivos e reação positiva ao teste IDGA mostrou-se presente apenas nos machos que apresentavam sinais clínicos $(p<0,05)$. Numa simulação, onde o número de animais foi duplicado, mantendo-se as mesmas características da amostra estudada, observou-se que os animais com sinais clínicos apresentavam uma taxa de positividade aproximadamente quatro vezes maior que os animais clinicamente sadios, confirmando a associação de histórico de infertilidade e distúrbios reprodutivos com anticorpos antiBrucella canis nos machos. Nas fêmeas, essa associação não foi observada, talvez por não ser a Brucella canis o principal microrganismo envolvido nos distúrbios reprodutivos nesses animais, já que outros agentes infecciosos e parasitários podem causar abortos, infertilidade, entre outras patologias do sistema reprodutor (Carmichael e Greene, 1993).

Ao se analisar as variáveis faixa etária, sexo e raça observouse que apenas a raça apresentou associação significativa com a soropositividade para infecção por B. canis (Tabela 2). Pesquisas realizadas por Azevedo et al. (2003); Cavalcanti et al. (2006) não demonstraram associação significativa entre a variável raça e soropositividade para $B$. canis.

Não se observou associação significativa entre infecção e faixa etária dos animais estudados. De acordo com revisão realizada por Azevedo et al. (2003) a maturidade sexual e conseqüente cobertura, bem como maior possibilidade de contato entre animais infectados pode ajudar a disseminação da doença. Entretanto, animais impúberes podem adquirir a infecção, onde normalmente a manifestação clínica é apenas uma linfadenopatia uni ou bilateral. No entanto, os sinais clínicos reprodutivos só se manifestam após a puberdade.

\begin{tabular}{llcccc}
\hline Variáveis & \multicolumn{1}{c}{ Descrição } & Positivo & Negativo & OR (IC 95\%) & Valor de $\boldsymbol{p}$ \\
\hline Faixa etária & 1 a 2 anos & - & 22 & $0,00(0,00$ a 7,58) & 0,426 \\
& Acima de 2 anos & 3 & 65 & & \\
Total & & 3 & 87 & & \\
Sexo & Macho & 1 & 27 & $1,11(0,02$ a 22,18) & 0,678 \\
& Fêmea & 2 & 60 & & \\
Total & & 3 & 87 & & \\
& & & & & \\
Raça & Com raça definida & 1 & 80 & $0,04(0,00$ a 1,01) & $0,025^{*}$ \\
& Sem raça definida & 2 & 7 & & \\
Total & & 3 & 87 & & \\
\hline
\end{tabular}

OR - odds ratio; *Associação significativa a $5 \%$

Nesse estudo o sexo não apresentou diferença significativa concordando com os resultados obtidos por Cavalcanti et al. (2006). De acordo com esses autores não há predisposição quanto ao sexo, estando machos e fêmeas expostos de uma forma semelhante ao risco de infecção.

\section{Conclusão}

Os resultados obtidos neste estudo demonstram que a infecção por Brucella canis ocorre na cidade de Maceió, AL e que medidas de controle e profilaxia devem ser empregadas para evitar a disseminação do agente para criatórios livres da doença. 


\section{Referências}

ACHA, P.N.; SZYFRES, B. Zoonosis y enfermidades transmissibles comunes al hombre y a los animales. Whashington: Organización Panamericana de la Salud, 1989.503 p.

ALMEIDA, A.C. et al. Soroepidemiologia da brucelose canina causada por Brucella canis e Brucella abortus na cidade de Alfenas, MG. Arquivo Brasileiro de Medicina Veterinária e Zootecnia, v. 56, n. 2, p. 275-276, 2004.

ALTON, G.G. et al. Techniques for the brucellosis laboratory. Paris: INRA, 1988. $190 \mathrm{p}$.

AZEVEDO, S.S. et al. Inquérito sorológico e fatores de risco para a brucelose por Brucella canis em cães do município de Santana de Parnaíba, Estado de São Paulo. Pesquisa Veterinária Brasileira, v. 23, n. 4, p. 156-160, 2003.

AZEVEDO, S.S. Brucelose por Brucella canis em cães do Município de Santana de Parnaíba - SP, Brasil. Inquérito sorológico, fatores de risco e comparação de testes diagnósticos. 2002. 100 p. Dissertação (Mestrado em Epidemiologia Experimental e Aplicada às Zoonoses) Faculdade de Medicina Veterinária e Zootecnia da Universidade de São Paulo, São Paulo.

BERTHELOT, X.; BASTUJI, B.G. A brucelose do cão. A Hora Veterinária, n. 92, p. 47-50, 1996.

CARMICHAEL, L.E.; KENNEY, R.M. Canine abortion caused by Brucella canis. Journal of American Veterinary Medical Association, v. 152, p. 605-616, 1968.

CARMICHAEL, L.E.; KENNEY, R.M. Canine brucellosis: the clinical disease, pathogenesis and immune response. Journal of American Veterinary Medical Association, v. 156, n. 12, p. 1726-1734, 1970.

CARMICHAEL, L.E.; GREENE, C.E. Brucellosis canina. In: CARMICHAEL, L.E.; GREENE, C.E. Enfermedades Infecciosas Perros y Gatos. México, Interamericana: Mcgraw - Hill, 1993. p. 604-615.

CAVALCANTI, L.A. et al. Pesquisa de anticorpos anti-Brucella canis em cães provenientes da região metropolitana de Salvador. Revista Brasileira de Saúde e Produção Animal, v. 7, n. 2, p. 176-180, 2006. CORTES, V.A et al. Reações sorológicas para Brucella canis em cães errantes capturados na proximidade de parques públicos, reservas florestais e em áreas periféricas do município de São Paulo. Revista da Faculdade de Medicina Veterinária e Zootecnia da Universidade de São Paulo, v. 25, n. 1, p. 101-107, 1988.

DEAN, A.G. et al. Epi Info, Version 6.01: A Word Processing, Database and Statistics Program for epidemiology on Microcomputers. Atlanta: Centers for Disease Control and Prevention, 1994.

ETTINGER, S.J.; FELDMAN, E.C. Tratado de Medicina Interna Veterinária - Moléstia do cão e do gato. São Paulo: Manole, 1997. 2256 p.

GODOY, A.M. et al. Isolamento de Brucella canis em Minas Gerais, Brasil. Arquivos da Escola de Veterinária da Universidade Federal de Minas Gerais, v. 29, n. 1, p. 35-42, 1977.
GOMES, M.J.P. etal. Brucella canis: Isolamento em um cão com epididimite e orquite - relato de caso. Clínica Veterinária, n. 18, p. 17-20, 1999.

GREENE, C.E.; GEORGE, L.W. Canine brucellosis. In: GREENE, C. E. Clinical Microbiology and Infectious Diseases of Dog and Cat. Philadelphia: 1984. p. 646-662.

JOHNSON, C.A.; WALKER, R.D. Clinical signs and diagnosis of Brucella canis infection. The Compendium on Continuing Education for the Practicing Veterinarian. Small Animal, v. 14, n. 6, p. 763-772, 1992.

KEID, L.B. Diagnóstico da brucelose canina por Brucella canis. Correlação entre exames clínicos e laboratoriais: imunodifusão em gel de ágar, imunodifusão em gel de agar com emprego do 2mercaptoetanol, cultivo e reação em cadeia de polimerase. 2001. 96 p. Dissertação (Mestrado em Reprodução Animal) - Faculdade de Medicina Veterinária e Zootecnia da Universidade de São Paulo, São Paulo.

LARSSON, M.H.M.A. et al. Canine brucellosis in São Paulo: serologic survey of kennel and stray dogs. International Journal Zoonoses, v. 8, p. 85-90, 1981.

MAIA, G.R. et al. Prevalência da brucelose canina nas Cidades do Rio de Janeiro e Niterói - RJ. Revista Brasileira de Reprodução Animal, v. 23, p. 425-427, 1999.

MINHARRO, S. Diagnóstico da brucelose canina: dificuldades e estratégias. Revista Brasileira de Reprodução Animal, v. 29, n. 3/4, p.167-173, 2005.

MORAES, C.C.G. et al. Prevalência da brucelose canina na Microrregião da Serra de Botucatu, São Paulo, Brasil. Arquivos do Instituto Biológico, v. 69, n. 2, p. 7-10, 2002.

NELSON, R.W.; COUTO, C.G. Fundamentos de Medicina Interna de Pequenos Animais. Rio de Janeiro: Guanabara-Koogan, 1992. 737 p. NICOLETTI, P.; CHASE, A. Avaliação dos métodos de diagnóstico da infecção por Brucella canis em cães. Cães e Gatos, p. 21-23, 1988. NUMERIANO, A.K.M. et al. Pesquisa de aglutininas anti-Brucella canis em cães errantes e domiciliados do Município de Recife - PE. In: CONGRESSO DE INICIAÇÃO CIENTÍFICA DA UFRPE, 10., Recife, PE. Anais... Recife: 1999. p. 215-216.

SOUZA, L.A. et al. Prevalência de infecção por Brucella canis em Belo Horizonte - MG. Revista Brasileira de Medicina Veterinária; v. 24, n. 3, 2002.

TRUSFIELD, M. Veterinary epidemiology. London: Butterworth, 1986, $273 p$.

VARGAS, A.C. et al. Brucelose canina: relato de um caso. Ciência Rural, v. 26, n. 12, p. 305-308, 1996.

WALD, V.B.; FERNANDES, J.C.T. Sorologia da brucelose canina no Município de Porto Alegre, RS. Arquivos da Faculdade de Veterinária da Universidade do Rio Grande do Sul, v. 4/5, p. 92-95, 1976/1977. 